\title{
A Computational Group Theory Study of Isotropic Subgroups Is Obtained
}

\author{
Un-Son Jong, Song-Won Kim, Ju-Myong Song, Jin-Ho Kim \\ Kim Hyong Jik University of education, Pyongyang, D.P.R.of Korea
}

\begin{abstract}
We established an algorithm to obtain the isotropy subgroup by using the channels of phase transition and developed the program.Using this algorith, we obtained precisely Landau symmetric phases of 230 space groups.
\end{abstract}

Keywords: isotropy subgroup, Landau symmetric phase, algorithm

\section{Introduction}

H. T. Stokes ${ }^{[1]}$ obtained 4777 irreps (irreducible representations) corresponding 80 Lifshitz stars using their rep table of Ref. [3] and classified into 132 inequivalent images. And he generated the isotropy subgroups of the 230 crystallographic space groups by algebraic method ${ }^{[6]}$.

In Ref. [2] 4834 irreps corresponding 80 Lifshitz stars were obtained by using the irrep tableof Ref. [4] and classified into 132 in equivalent images. Besides a general algorithm and the database for generating the isotropy subgroups of the 230crystallographic space groups were presented.

When the second order phase transition is taken place in the different crystals, the symme-try of the system is broken. This is well known ${ }^{[5]}$.

Now let the parent phase is characterized by the symmetry group $G$ that leaves invariant the distribution function $\rho_{0}(\vec{r})$ of charge density of the system.

$G$ is one of the 230 space groups.

When the second order phase transition is taken place, the symmetry of the new phase might become low. Because some symmetric elements are vanished since the state is chan-ged continuously.

Then the new phase will be determined by the subgroup $G_{\alpha}$ of the space group $G$.

$$
G_{\alpha} \subset G
$$

When the spontaneous symmetry breaking is occured, the distribution function of charge density $\rho_{0}(\vec{r})$ changes as $\delta \rho(\boldsymbol{r})$. The low symmetry phase is described by the distribution function $\rho(\boldsymbol{r})$ of charge density.

$$
\rho(\boldsymbol{r})=\rho_{0}(\boldsymbol{r})+\delta \rho(\boldsymbol{r})
$$

The symmetric elements of $\rho(\boldsymbol{r})$ or $\delta \rho(\boldsymbol{r})$ form the space group $G_{\alpha}$. That is,

$$
g \in G_{\alpha} . \quad \hat{g} \delta \rho(\boldsymbol{r})=\delta \rho(\boldsymbol{r})
$$

When the phase transition is taken place according to an irrep of crystal symmetry group $G$,we can write the change $\delta \rho$ in the distribution function of charge density of the new phaseas follows:

$$
\delta \rho(\boldsymbol{r})=\sum_{\alpha=1}^{m} \sum_{\beta=1}^{l} C_{j \beta}^{(\alpha)} \varphi_{j \beta}^{(\alpha)}(\boldsymbol{r})
$$

where $\alpha$ is the number of arms of the wave vector star and $\beta$ is the number of basic functions of the $\mathrm{j}^{\text {th }}$ irreducible representation of the wave vector group $G_{k(\alpha)}$ which is related to an arm $\boldsymbol{k}(\alpha)$ of star $\boldsymbol{k}$.

The set of the expansion coefficients $C_{j \beta}^{(\alpha)}$ may be sorted into two. One is $\{\eta\}$ (critical order parameter), the set of the coefficients that describes the symmetry breaking directly, and another is $\left\{C_{j, a}^{k}\right\}$ (noncritical order parameter), the set of the coefficients that doesn't influence the symmetry breaking,but describes thephysical properties.

In this paper, we study thespontaneous symmetry breaking by the theory of symmetry, so we consider the critical order parameter.

After the Landau theory, the phase transitiontakes place according to a certain irrep.

$$
\delta \rho(\boldsymbol{r})=\sum_{k, j, a} \eta_{j, a}^{k} \varphi_{j, a}^{k}(\boldsymbol{r})
$$

Because the set of $\varphi_{i}(\boldsymbol{r})$ consists of the completing system in the formula (5), the setof $\eta_{m}$ consists of the base of the representation $T_{i}$ and $\left\{\eta_{i}\right\}$ becomes the irreducible order parameter characterizing the possible low symmetry phase which define in the phase transition.

The set of the invariant vector of the I-Group(Image group)is the set of the order parameters characterizing the possible low symmetry phase in the phase transition by a 
certain representation $T_{i}$ of the space group $G$ and is equivalent to the solutions of the state equation.

All the possible low symmetry phases that are obtained by the set of the invariant vectorsof the I-Group when the space group $G_{0}$ of parent phase and the representation $T$ that induces the phase transition are given, is called an isotropy subgroup.

In the study we established the algorithm for obtaining the isotropy subgroup, made the database and develolped the program.

\section{Algorithm for obtaining the isotropy subgroup}

1) Procedure for obtaining the isotropy subgroup

If the space group $G_{0}$ of the parent phase and the wave vector $\vec{k}$ were given, the procedure for finding the space group $G_{\alpha}$ of the low symmetry phase induced by a certain representation of $G_{0}$ is as follows.

(1) Determining the space group $G_{\alpha}$ of the low symmetry phase by the symmetric elements that map the order parameter $\vec{\eta}$ invariant. $G_{\alpha}$ is as follows.

$G_{\alpha}=\left\{\left(h_{1}^{\alpha} \mid 0\right)+\cdots+\left(h_{i}^{\alpha} \mid \tau_{i}^{\alpha}+t_{i}^{\alpha}\right)+\cdots+\left(h_{g}^{\alpha} \mid \tau_{g}^{\alpha}+t_{g}^{\alpha}\right)\right\} \operatorname{ker} G_{\mathrm{t}}$

$G_{\alpha}^{\mathrm{t}}=\left\{\left(h_{1}^{\alpha} \mid t_{1}\right)+\left(h_{1}^{\alpha} \mid t_{2}\right)+\cdots+\left(h_{i}^{\alpha} \mid t_{g}\right)\right\} \operatorname{ker} G_{\mathrm{t}}$

We find the elements corresponding with the point group $G_{\alpha}^{0}$ by $G_{\alpha}^{0}=G_{\alpha} / G_{\alpha}^{\mathrm{t}}$

Where $\left(h_{1}^{\alpha} \mid 0\right),,\left(h_{g}^{\alpha} \mid \vec{\tau}_{g}^{\alpha}+\vec{t}_{g}^{\alpha}\right)$ are the representative coset elements by $\operatorname{ker} G_{t}$ characterizing the Landau symmetry phase $G_{\alpha},\left(h_{1}^{\alpha} \mid \vec{t}_{1}^{\alpha}\right),,\left(h_{g}^{\alpha} \mid \vec{t}_{g}^{\alpha}\right)$ are the representative coset elements of $G_{\alpha}^{\mathrm{t}}$ by $\operatorname{ker} G_{t}$.

(2) Finding the starline $k_{i}$ which takes part in the phase transition which satisfies

$\exp \left(-i k_{i} t_{g}\right)=1$ by the given star $\vec{k}$ and the representative coset elements $\left\{\vec{t}_{g}\right\}$ of $G_{\alpha}^{\mathrm{t}}$

and determines the star channel $\lfloor\vec{k}\rfloor$. The change of the volume $p$ is $\left[G_{f}^{t}\right] /\left[G_{\alpha \mathrm{f}}^{t}\right]$.

(3) Obtaining the phase transition channel. ${ }^{[6]}$

First, we must precisely know the crystal system of the lattice.

To select the crystal system means that the point group of the possible lower symmetry phase is determined in the view of the group theory. Meanwhile, each Bravais lattices must be invariant to translation group because the crystal has the translational symmetry.
The translation group is related to various star channels of the wave vector star and the point group of lattices is isomorphic to the channel point group $G_{[k]}^{f}=G_{[k]}^{0}=G_{[k]} / T_{[k]}$. After all, the point groups of the possible lower symmetry during the spontaneous symmetry breaking of the crystal are isomorphic to the subgroups of the point group $G_{[k]}^{0}$ of the star channel associated with a given wave vector star. So, we found out the crystal system of the possible Bravais lattice during the translational symmetry breaking by means of the star channel point group are its subgroups.

Second, we must exactly know the vector orientation of the Bravais lattice.

We found it out by means of the translation group of the star channel and its kernel of the representation.

The crystal lattice of the new phase is characterized by translation vectors $\boldsymbol{t}_{\mathrm{D}}$ satisfying the equation $\exp \left(i \boldsymbol{k}_{\mathrm{\alpha}} \boldsymbol{t}_{\mathrm{D}}\right)=1$ with respect to the given wave vector star channel $\left[\boldsymbol{k}_{\alpha}\right]$. The set of these translation vectors belongs to representation kernel $\operatorname{Ker}(\boldsymbol{t})$ which are invariant subgroups of the translation group $T_{[k]}$ of the star channel.Among these translation vectors $\boldsymbol{t}_{\mathrm{D}}$, the vectors $\boldsymbol{d}_{[k]}=\left\{d_{1}, d_{2}, d_{3}\right\}$ having the shortest length become the vectors of Bravais cell of the lower symmetry phase. The volume change of a unit cell during the phase translation is expressed as $n=T_{[k]} / \operatorname{ker} \Gamma(t)$

Third, we must find out the possible Bravais lattice types during the phase transition.

We found out the various subgroups of the star channel poin $t$ group and obtained the Bravais lattice types $\boldsymbol{d}_{[k] \mathrm{s}}$ that were invariant to them. We just defin e the Bravais lattice types thatare invariant to the subgroups $G_{[k] s}^{0}$

of the star channel point group as lattice channel andexpress like $\boldsymbol{d}_{[k] \mathrm{s}}(\mathrm{s}=0,1, \mathrm{~s})$. Generally one wave vector star channel $\mathrm{h}$ as several lattice channels. So, we can define the phase trans ition channel as the sum of the star channel and its lattice ch annels.

\section{Phase transition channel=star channel + lattice channel}

Conveniently, the phase transition channel is expressed as $P_{[k]}^{d_{[k] s}}$.Here $i$ is the number of the wave vector star channel $[\boldsymbol{k}], s$ is the number of lattice channel $\boldsymbol{d}_{[k] \mathrm{s}}$ of the given star channel $[k], j$ is the number of various orientations of the translation vectors in a lattice channel.

The phase transition $P_{[k]}^{d_{[k] s}}$ means that the lower symmetry phase has the $j$ th lattice types of the sth lattice channel when the phase transition occurs along the $i$ th star channel in crystal.

Next we must find the transformation matrix A based on the given phase transition chan-nel.

(4) Coinciding with the rotation and translation parts of the higher symmetry phase and the lower symmetry phase by the following formulas. 


$$
\begin{gathered}
D^{\alpha}\left(h_{k}^{\alpha}\right)=A^{-1} D\left(h_{k}\right) A \\
S=C^{-1} A^{-1} B
\end{gathered}
$$

In the formula (7) matrix $D\left(h_{k}\right)$ is the coordinate transition matrix of the rotational part $h_{k}$ of the space group element of the higher symmetry phase and matrix $D\left(h_{k}^{\alpha}\right)$ is the coordinate transition matrix of the rotational part $h_{k}^{\alpha}$ of the space group element of the lower symmetry phase.

(5) Coinciding with the obtained space group $G_{\alpha}$ with the standard space group $G_{\beta}$.

$$
\vec{\tau}_{i}^{\alpha}-\vec{V}+h_{i}^{\alpha} \vec{V}=\vec{\tau}_{i}^{\beta} \pm n_{i j} \vec{t}
$$

If the orgin doesn't move, choose the new transition matrix associated with the star channelpointed in the phase transition channel, the change of the volume, the pointgroup of the star channel and classify the standard space group.In the figure 1 we showed the calculation flow chart for finding the isotropy subgroup.

2) Algorithm for obtaining the isotropy subgroup

Algorithm is consist of two stages, One is the algorithm for finding the point group of the low symmetry phase and theother is the algorithm which coincides with the rotational par-ts and the translation parts of the elements of the space group of the higher symmetry phase and the lower symmetry phase.

(1) Finding the symmetry element $\hat{G}: \hat{G} \vec{\eta}=\vec{\eta}$

The full irrep matrices of the zero block type corresponding the symmetric element $\hat{G}$.act on the invariant vector $\vec{\eta}$.Because the component of the invariant vector $\vec{\eta}$ is indicated as $(a+b \vec{i}) \eta_{n}$, find $\hat{G}$ which coincides with the coefficients and the number of $\eta$.The full irrep considering translation act on the the invariant vector $\vec{\eta}$ and repeat the above process.

(2) Abstracting the rotation elements of the spacegroup which leave $\vec{\eta}$ invariant

If the space group of the parent symmetry phase belongs to any one of the triclinic, monoclinic, orthorhombic, tetragonal, cubic system, we must find the numbers of the symmetry elements in the table 《Cubicelement》.If the space group of the parent symmetry phase belongs to hexagonal system, we must find the numbers of the symmetry elements in the table

《Hexalement》.If the space group of the parent symmetry phase belongs to Rhombhedral system, we must find the numbers of the symmetry elements in the table

《Rhombement》, we must decide the point group of the lower symmetry phase by using the symmetry elements and their number.

(3) Finding the phase transition channel.

We must make the table 《star channel》, table 《lattice channel》indicating the elements of the point group abstracted in (1), the point group of the low symmetry phase, the change of the volume and we must abstract the symmetry transition matrices.

(4) Coinciding with the rotational parts of the high symmetry phase and the low symmetry phase. Use the formula $D\left(h_{k}^{\alpha}\right)=A^{-1} D\left(h_{k}\right) A$. Then the rotational part $h_{k}$ of the higher symmetry phase becomes the rotational part $h_{k}^{\alpha}$ of the lower symmetry phase.

(5) Coinciding with the translational parts of the high symmetry phase and the low symmetry phase. Use the formula $S=C^{-1} A^{-1} B$.After all the the translational part $\vec{\tau}_{i}$ of the high symmetry phase becomes the low symmetry phase $\vec{\tau}_{i}^{\alpha}$.

Finding the standard space group, moving the the origin, by means of the formula of theorigin displacement, $\vec{\tau}_{i}^{\alpha}-\vec{V}+h_{i}^{\alpha} \vec{V}=\vec{\tau}_{i}^{\beta} \pm n_{i j} \vec{t}$

Where $\vec{V}$ is the coordinate $(x, y, z)$ of the origin displacement. $h_{i}^{\alpha}$ is the data of the T(h) field of the table 《ActionVector》 indicating the coordinatetransition matrix of the low symmetry. $\vec{\tau}_{i}^{\alpha}$ is the translation vector of the space group of the low symmetry phase and $\vec{\tau}_{i}^{\beta}$ is the improper translation vector of the rotational elements presented in the paper [2].We showed the flow chart for obtainingthe isotropy subgroup in figure 1. 


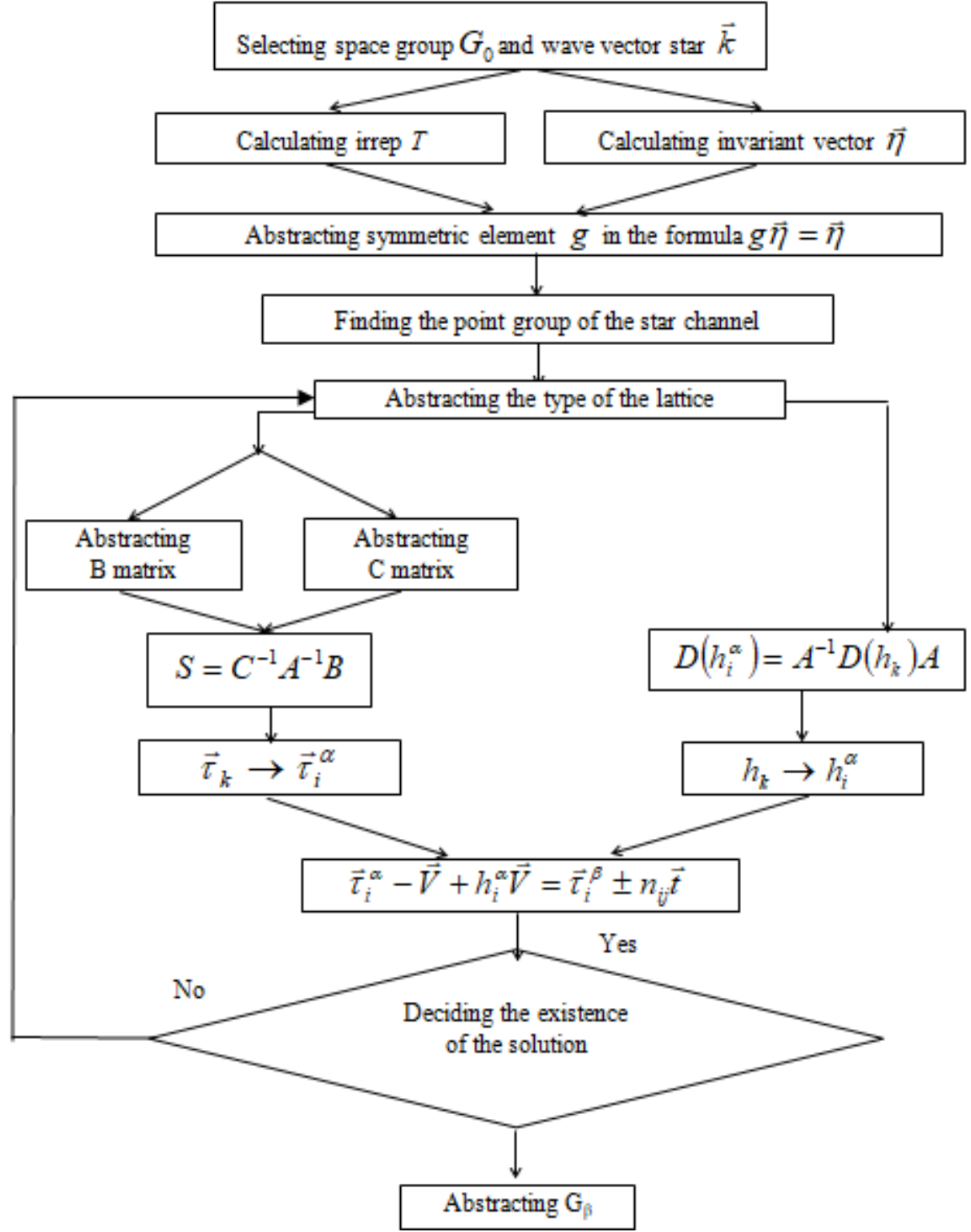

Figure 1: The flow chart for obtaining the isotropy subgroup

\section{Design and construction of the database for obtaining the isotropy subgroup}

To obtain the isotropy subgroup of the 230 space group from the set of the invariant vectors of the I-Group expressing the information of the 230 crystallographic space group and cystallographic lattice we need the information of the improper translation vector corresponding to the rotational part of the element of the space group, the information of the basic translation vectors of the 14 Bravais lattice and the volume of the unit cell, the information of the full symmetric point group, the information of the projective representat-ion matrix, the information of the correspondence relationship between the wavevector and projective representation, the information of the multiplication table of the rotational elements of the cubic system and hexagonal system, the information of the coordinate transition rule of the rotation, the information of the invariant charactersof the I-Group (the information of the correspondence relationship between the I-Group and irrep, the inf-ormation of the set of the invariant vectors), the information of the point group for obtaini-ng the star channel point group, the information of the star channel and the lattice channel.

From now on, the database <ISOSUBGROUP.mdb> is added the 11 tables except the database <FIRP.mdb> to find the irrep of the wave vector star group

The database <FIRP.mdb $>$ consists of the following tables.

1) $<$ SpaceGroup $>$ table- the information of the elements of the space group

2) <Wavevector > table - the information of the wave vector

3) <14Lattice> table - the information of the 14 Bravais lattice

4) $\langle\mathrm{T}(\mathrm{N})\rangle$ table - the information of the projective representation matrix

5) <Wavevector> table - the information of the correspondence relationship between the wavevector and projective representation

6) <Element> table - the information of the the elements of the point group 
7) <Cubic operation> table - the information of the multiplication table of the rotation ele-ments of the cubic system

8) < Hexaoperation> table - the information of the multiplication table of the rotation ele-ments of the hexagonal system

9) <Action vector> table - the information of the coordinate transition rule corresponding the rotation elements

The added tables are as follows.

1) <Cubicelement $>$ table - the information of the elements of the cubic system

2) <Hexaelement $>$ table - the information of the elements of the hexagonal system

3) <Rhombelement> table - the information of the elements of the rhombohedral system

4) <Pointgroup > table - the information of the point group

5) $\langle$ tp $>$ table - the information of the correspondence relationship of the element of the point group between phase transition channel and star channel

6) $<$ Bravais $>$ table - the information of the lattice channel

7) $<$ B_C $>$ table -- the information of the correspondence relationship of the basic translation vector between the primitive cell and the Bravais cell

8) <Star> table - the information of the correspondence relationship between the star cha-nnel and phase transition channel

9) <aelement> table - the information of the elements of the point group

10) $<$ Rep-IG $>$ table - the information of the correspondence relationship between the I-group and irreducible representation

11) <Invarvector> table - the information of the invariant vector

The structure parts of the added tables are as follows.

Table 1: <Cubicelement>

\begin{tabular}{|c|c|c|c|}
\hline Field name & Field type & size & Description \\
\hline ID & AutoNumber & & The number of the rotation element \\
Element & Text & 5 & Symmetry element expression symbol \\
D(h) & Text & 50 & the coordinate transition matrix \\
\hline
\end{tabular}

Table 2: 〈Pointgroup>

\begin{tabular}{|c|c|c|c|}
\hline Field name & Field type & size & description \\
\hline ID & AutoNumber & & The number of the Pointgroup \\
Cry_ID & Number & Byte & The number of the Crystal system \\
Sym & Text & 5 & Pointgroup expression symbol \\
Sub & Text & 5 & Subscript \\
$1 \sim 8$ & Number & byte & The number of the symmetry element \\
\hline
\end{tabular}

Table 3: $\langle\mathrm{tp}>$

\begin{tabular}{|c|c|c|c|}
\hline $\begin{array}{c}\text { Field } \\
\text { name }\end{array}$ & $\begin{array}{c}\text { Field } \\
\text { type }\end{array}$ & size & description \\
\hline Cry_ID & Number & Byte & The number of the Crystal system \\
Sub1 & Text & 5 & Subscript of the phase transition channel \\
Sup & Text & 5 & $\mathrm{P}_{\mathrm{i}}^{\mathrm{j}}$ \\
Sym & Text & 5 & Supscript of the phase transition channel \\
Sub & Text & 5 & $\mathrm{P}_{\mathrm{i}}^{\mathrm{j}}$ \\
$\mathrm{H}$ & Text & 150 & The part except of the subscript in the \\
$\mathrm{P}$ & Number & byte & symbol \\
$\mathrm{m}$ & Text & 20 & subscipt of the symbol of the point group \\
\hline
\end{tabular}

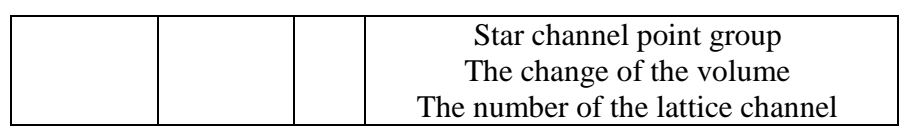

Table 4: $\left\langle\mathrm{B} \_\mathrm{C}\right\rangle$

\begin{tabular}{|c|c|c|c|}
\hline $\begin{array}{c}\text { Field } \\
\text { name }\end{array}$ & Field type & size & description \\
\hline $\begin{array}{c}\text { ID } \\
\text { Latt_ID } \\
\text { C }\end{array}$ & $\begin{array}{c}\text { Autonumber } \\
\text { Number } \\
\text { Text }\end{array}$ & $\begin{array}{c}\text { Byte } \\
50\end{array}$ & $\begin{array}{c}\text { The number of the Bravais lattice } \\
\text { The number of the lattice type of } \\
\text { the low symmetry phase } \\
\text { the relation matrix of the basic } \\
\text { translation }\end{array}$ \\
vector and the edge vector
\end{tabular}

Table 5: <Bravais>

\begin{tabular}{|c|c|c|c|}
\hline Field name & Field type & size & description \\
\hline Cry_ID & Number & Byte & The number of the Crystal system \\
Sub & Text & 5 & subscipt of the symbol $\mathrm{P}_{\mathrm{i}}^{\mathrm{j}}$ \\
Sup & $"$ & $\prime \prime$ & supscipt of the symbol $\mathrm{P}_{\mathrm{i}}^{\mathrm{j}}$ \\
Latt_ID & number & byte & The number of the lattice type \\
A & Text & 50 & the matrix of the symmetry transition \\
$\mathrm{m}$ & Text & 2 & the numberof the lattice channel \\
& & & corresponding A matrix \\
\hline
\end{tabular}

Table 6: <star>

\begin{tabular}{|c|c|c|c|}
\hline Field name & Field type & size & description \\
\hline Cry_ID & Number & Byte & The number of the Crystal system \\
Star_kovalev & Number & Byte & Kovalev star number \\
StL & Text & 10 & Srar line number \\
Sub1 & Text & 20 & subscipt of the symbol $\mathrm{P}_{\mathrm{i}}^{\mathrm{j}}$ \\
sup & Number & byte & supscipt of the symbol $\mathrm{P}_{\mathrm{i}}^{\mathrm{j}}$ \\
\hline
\end{tabular}

Table 7: <element>

\begin{tabular}{|c|c|c|c|}
\hline Field name & Field type & size & description \\
\hline SG_ID & Number & Byte & The number of the space group \\
Sup_ID & Number & Byte & supscipt of the space group symbol \\
Latt_ID & Number & Byte & The number of the lattice type \\
$\mathrm{h}_{\mathrm{i}}$ & Text & 50 & Improper translation vector \\
\hline
\end{tabular}

Table 8: 〈Rep_IG>

\begin{tabular}{|c|c|c|c|}
\hline Field name & Field type & Size & description \\
\hline SG_ID & Number & Byte & The number of the space group \\
Star_kovalev & Number & Byte & $\begin{array}{c}\text { Kovalev star number } \\
\text { IRP_ID }\end{array}$ \\
Number & Byte & $\begin{array}{c}\text { Irreducible representation number } \\
\text { I-group symbol }\end{array}$ \\
I_G & Text & 10 & \\
\hline
\end{tabular}

Table 9: <Invarvector>

\begin{tabular}{|c|c|c|c|}
\hline $\begin{array}{c}\text { Field } \\
\text { name }\end{array}$ & Field type & size & description \\
\hline ID & Autonumber & & Identification number \\
I_G & Text & 10 & I-Group symbol \\
Lv_ID & Number & byte & The number of the invariant vector \\
The coefficient of the invariant \\
$\mathrm{Ph}_{1}$ & Text & 20 & vector \\
$\mathrm{Ph}_{2}$ & Text & 20 & The number of the component $\eta_{\mathrm{n}}$ \\
\hline
\end{tabular}

The strucrture of the table <Hexaelement> and the table

《Rhombelement 》 is as table 2 .

The relationship of every tables is as infigure 2 . 


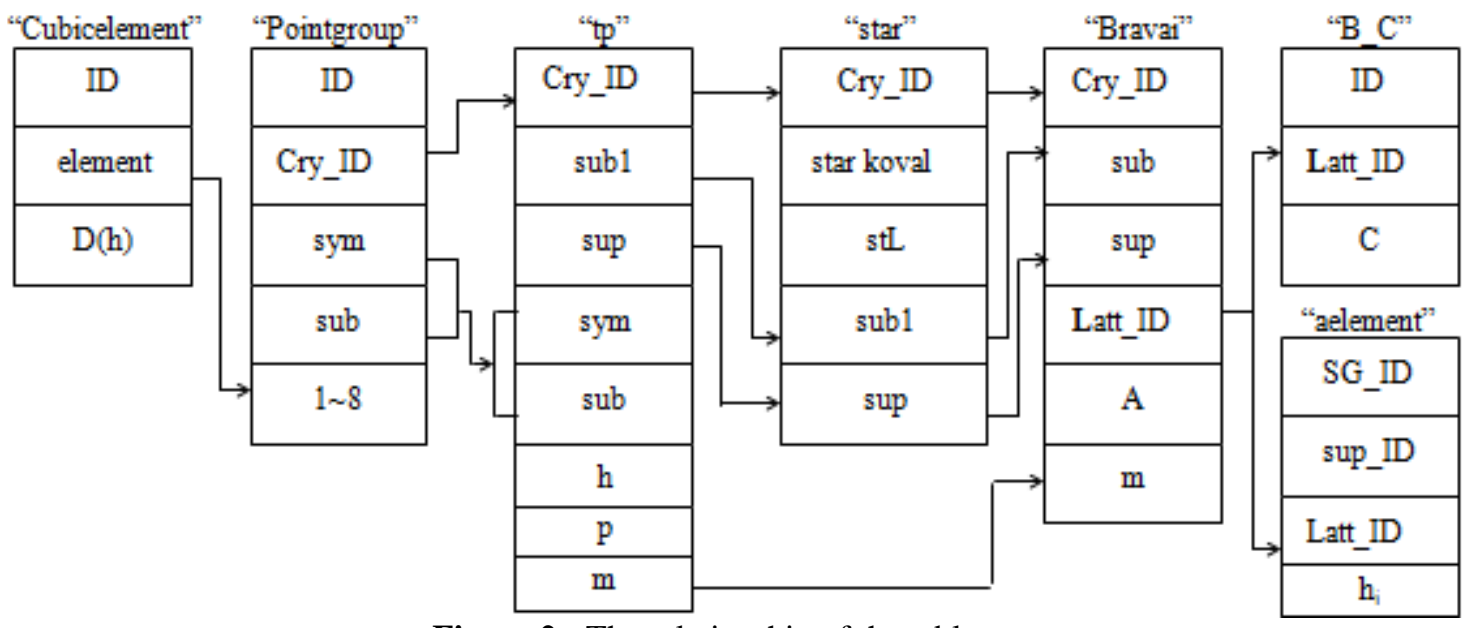

Figure 2: The relationship of the tables

We showed the algorithm for obtaining the isotropy subgroup in the figure 3.

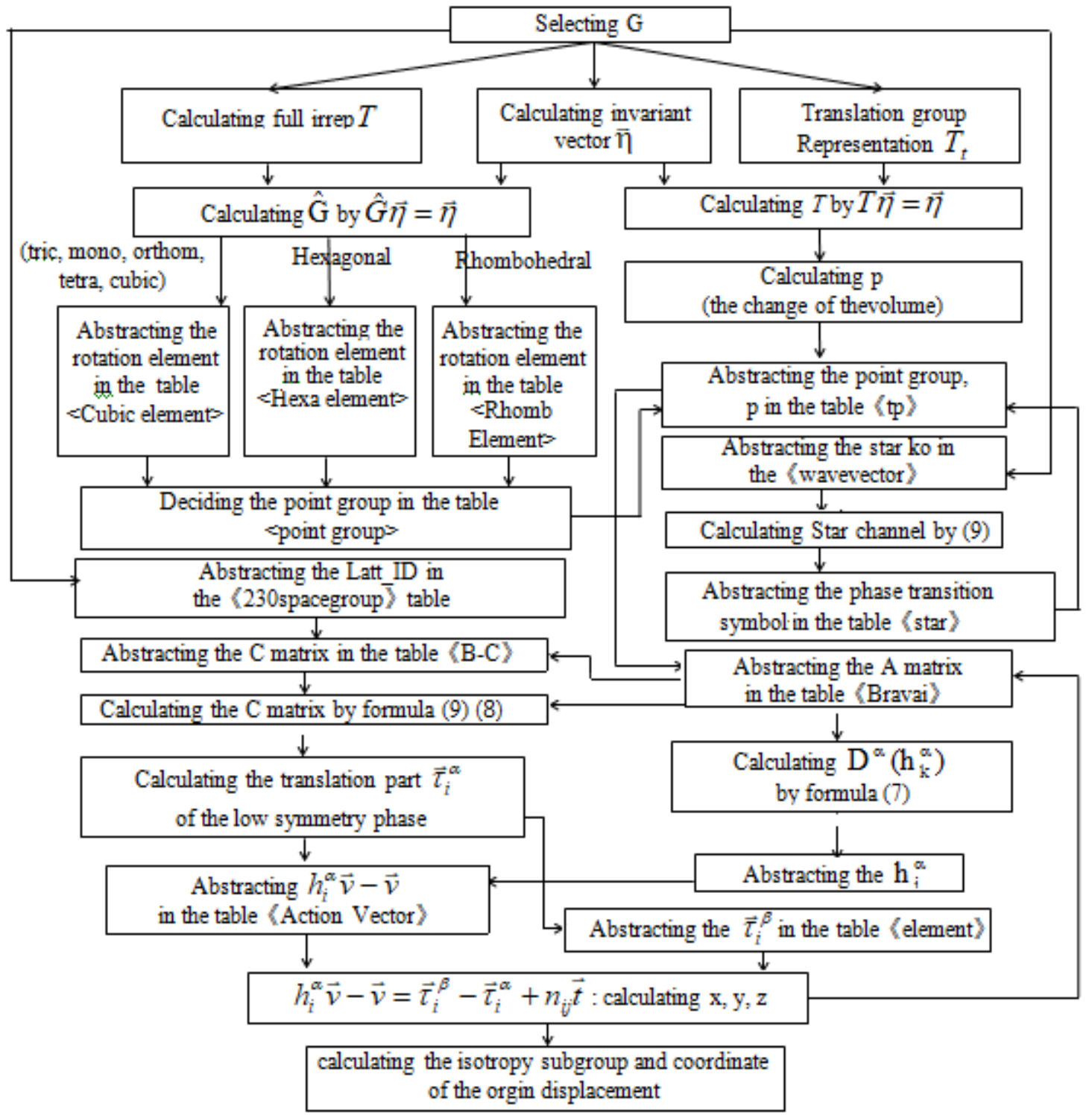

Figure 3: The algorithm for obtaining the isotropy subgroup 


\section{Comparison with the paper [6]}

Let's compare the method suggested In this paper using the algebraic method.[1]

In our paper we instituted the correspodence relationship by the phase transition channel in the condition that the lattice type and the transition matrix are given.

But in the algebraic method the symmetry phases were given.It finds the equivalent point group from the $G^{\prime}$ the low symmetry phase if the space group wasn't yet decided andselected the standard type $G^{\prime \prime}$ based on all the lattice types.

They institute the corresponding relationship $R_{i}^{\prime} \rightarrow R_{j}^{\prime \prime}$ of the rotation elements based on the arbitrarily selected space group $G^{\prime \prime}$. In the case that the standard type of the space grou-p isn't obtained selected the transition matrix A must be selected directly again, but the algebraic method finds the transition matrix S set up $R_{i}^{\prime} \rightarrow R_{j}^{\prime \prime}$, if the solution wasn't obtai-ned, the standard space group $G^{\prime \prime}$ was selected.

The method of finding the transition matrix $S$ solves the 9 equations of each point group operator $R_{i}$, it is to solve the solutions that all the $S_{k j}$ are the integers, $\operatorname{det} S=1$. When the isotropy subgroups of the 230 space groups corresponding with the symmetry point $\vec{k}$ are considered,by the number of $-2,-1,0,1,2\left|S_{k j}\right| \leq 2$ about the 9-m independent parameter $S_{k j}$ attempt the $5^{9-m}$ set of integer,but it didn't find of some $\left|S_{k j}\right|$. When we calculate the orgin displacement, in this paper we compare with the some space groups of the the lattice type because we already know the lattice type.But in the ref[7]they compare with the man-y space groups of all the lattice type (primitive, base-centered, face-centered, body-centered because the concrete lattice type is n't indicated they we move the orgin.

The new method presented in the paper find the transition matrix A directly based on thephase transitionchannel and can decide the isotropy subgroup correctlyin a short time.

\section{Conclusion}

In this paper we obtained all the isotropy subgroups of the 230 crystallographicspace groups by the invariant vectors charcterized by 132 Image groups.

The 669 full irreps of the 36 space groups of the cubic system generated the possible 5 202isotropy subgroups characterized of the invariant vectors of the 120 I-Groups.

The 1514 full irreps of the 68 space groups of the tetragonal system generated the possible 4107 isotropy subgroups characterized of the invariant vectors of the 26 I-Groups.

The 1029 full irreps of the 52 space groups of the rhombohedral and hexagonal system generated the possible 3337 isotropy subgroups characterized of theinvariant vectors of the 64 I-Groups.

The 1392 full irreps of the 59 space groups of the orthohombicsystem generated the possible 2304 isotropy subgroups characterized by the invariant vectors of the $10 \mathrm{I}-$ Groups.

The 206 full irreps of the 13 space groups of the monoclinicsystem generated the possible 265 isotropy subgroups characterized of the invariantvectors of the 4 IGroups 


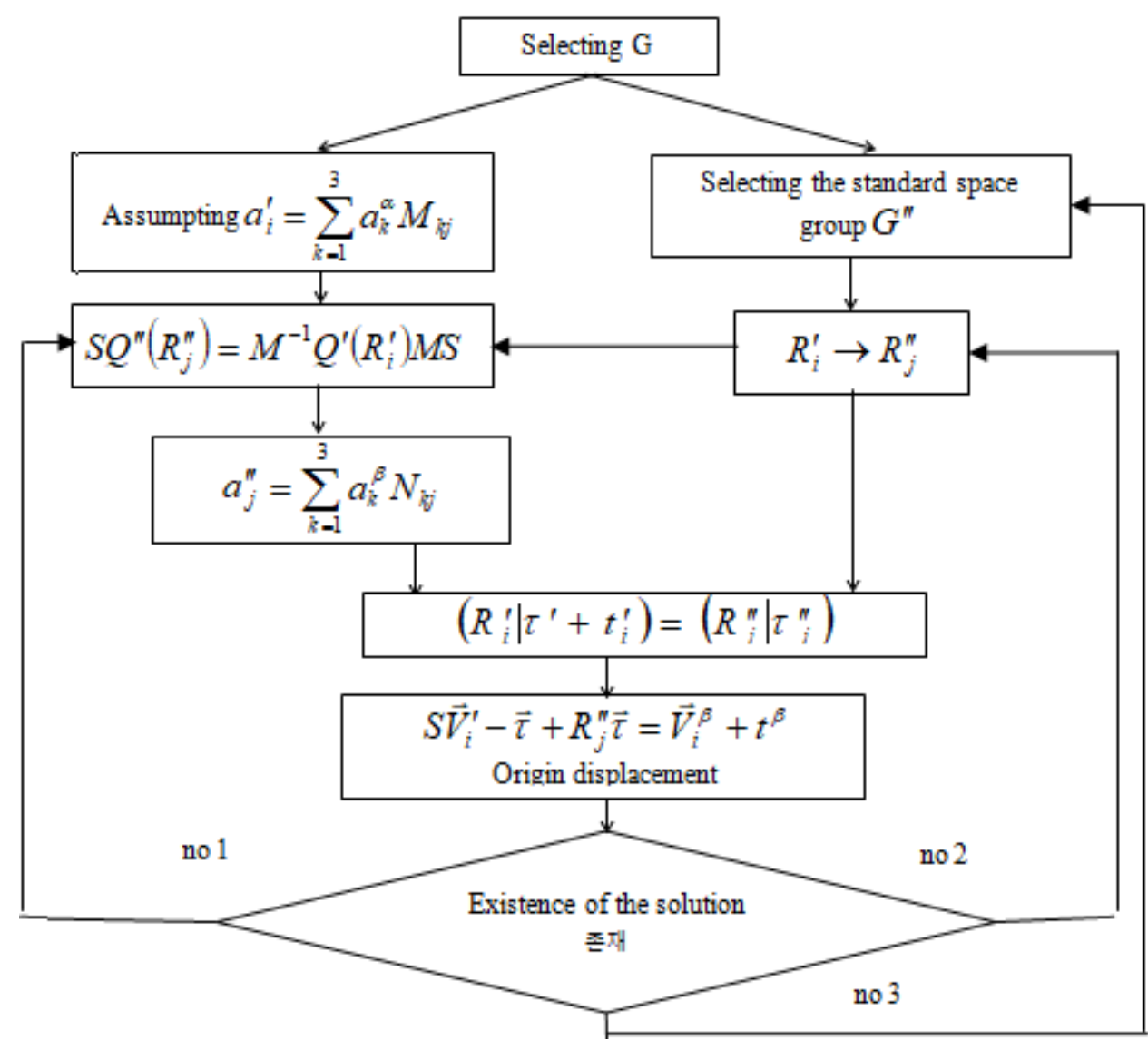

Figure 4: The flow chart for generating the isotropy subgroup by the algebraic method

\section{References}

[1] H.T.Stokes et al, Isotropy Subgroups of the 230 Crystallographic Space Groups, World Scientific,Singapore,380, 1988.

[2] Un Son Jong, et al. 《Study on Classification of Image Group》IJSR, vol 5 Issue 6, 424,2016.

[3] C.J.Bradley, A.P.Cracknell. The Mathematical Theory of Symmetry in Solids. 1972

[4] O. V. Kovalev, Representations of the Crystallographic Space Groups, Irreducible Representations, Induced Representations and Corepresentations. Gordon andBreach, Amstrerdam, 350, 1993.

[5] Л. Д. Ландау, идр. СтатистическаяФизика, М. Наука, 286. 2006.

[6] Il Hwan Kim, et al. 《Wave vector and Bravais Lattice associated symmetry》IJSR, vol 5 Issue 7,1338, 2016.

[7] H.T.Stokes, Isotropy.Tutorial http://www.physics.byu. edu/ stokesh/ isotropy. html. 2013. 